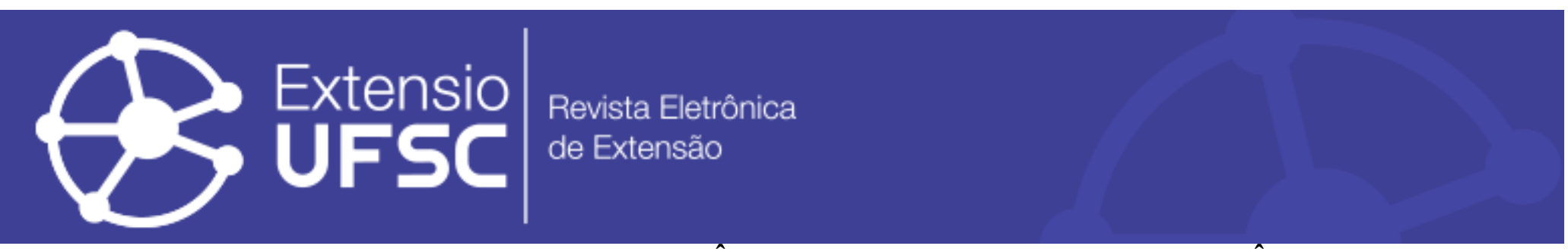

\title{
USO DE RADIOGRAFIAS PANORÂMICAS NO PROGRAMA DE ASSISTÊNCIA ODONTOLÓGICA A PACIENTES DE TRANSPLANTE DA UFMG
}

\author{
Maria Elisa Souza e Silva \\ Universidade Federal de Minas Gerais \\ mariaelisa1956@gmail.com \\ Luanne Priscila Pereira Avelar \\ Universidade Federal de Minas Gerais \\ luannepriscilla1@hotmail.com \\ Ellen Marise Castro Oliveira \\ Universidade Federal de Minas Gerais \\ elenmarisecastro@gmail.com
}

Tânia Mara Pimenta do Amaral Universidade Federal de Minas Gerais taniapamaral@gmail.com

Jéssica Alves de Borba Universidade Federal de Minas Gerais borba.jessica.alves@gmail.com

Lia Silva de Castilho

Resumo

Universidade Federal de Minas Gerais liasilvacastilho@gmail.com

A radiografia panorâmica é usada para a avaliação do estado dos dentes e osso dos maxilares, em uma visão global dessas estruturas de uma só vez. O objetivo deste estudo é caracterizar os pacientes e levantar aspectos radiográficos relevantes observados nas radiografias panorâmicas dos pacientes do Programa de Extensão Assistência Odontológica a Pacientes de Transplante da UFMG. Foram estudadas radiografias panorâmicas desde 2016. A análise estatística se deu por descrição dos resultados e análises bivariadas, empregando-se o teste do qui-quadrado e teste exato de Fischer, Mann-Whitney e KruskalWallis. Foram considerados significativos os resultados com $\mathrm{p}<0,05$. Em relação à faixa etária, foi observada uma associação significativa com a avaliação da raiz residual, nível ósseo e relação da cúpula alveolar. A radiografia panorâmica contribui para a compreensão de problemas, acelerando o estabelecimento do plano de tratamento, especialmente entre pacientes que estejam na fase pré-transplante de fígado, rins ou células hematopoiéticas.

Palavras-chave: Transplante de Fígado; Transplante de Células-Tronco Hematopoéticas; Transplante de Rim; Assistência Odontológica; Radiografia Panorâmica.

\section{USE OF PANORAMIC RADIOGRAPHS IN THE UFMG TRANSPLANT PATIENT DENTAL CARE PROGRAM}

\section{Abstract}

Panoramic radiography is used to assess the state of the teeth and bone in the jaws, in a global view of these structures at once. The objective of this study is to characterize the patients and to raise relevant radiographic aspects observed in the panoramic radiographs of the patients of the Extension Program Dental Assistance to Transplant Patients at UFMG. Panoramic radiographs have been studied since 2016. Statistical analysis was performed by describing the results and bivariate analyzes using the chi-square test and Fischer, Mann-Whitney and Kruskal-Wallis exact test. Results with $\mathrm{p}<0.05$ were considered significant. Regarding the age group, a significant association was observed with the assessment of the residual root, bone level and alveolar dome relationship. Panoramic radiography contributes to the understanding of problems by accelerating the establishment of the treatment plan, especially among patients who are in the pre-transplant phase of liver, kidneys or hematopoietic cells.

Key-words: Liver Transplantation; Hematopoietic Stem Cell Transplantation; Kidney Transplantation; Dental Care; Radiography, Panoramic.

\section{USO DE RADIOGRAFÍAS PANORÁMICAS EN EL PROGRAMA DE ASISTENCIA DENTAL PARA PACIENTES TRASPLANTADOS EN UFMG}

\section{Resumen}

La radiografía panorámica se utiliza para evaluar el estado de los dientes y el hueso de los maxilares, en una vista global de estas estructuras a la vez. El objetivo de este estudio es caracterizar a los pacientes y plantear aspectos radiográficos relevantes observados en las radiografías panorámicas de los pacientes del Programa de Extensión Asistencia Odontológica a Pacientes Trasplantados en la UFMG. Las radiografías panorámicas se estudian desde 2016. El análisis estadístico se realizó mediante la descripción de los resultados y los análisis bivariados mediante la prueba de chi-cuadrado y la prueba exacta de Fischer, Mann-Whitney y Kruskal-Wallis. Los resultados con $\mathrm{p}<0,05$ se consideraron significativos. En cuanto al grupo de edad, se observó una asociación significativa con la evaluación de la relación raíz residual, nivel óseo y cúpula alveolar. La radiografía panorámica contribuye a la comprensión de los problemas al acelerar el establecimiento del plan de tratamiento, especialmente entre los pacientes que se encuentran en la fase previa al trasplante de hígado, riñones o células hematopoyéticas.

Palabras llave: Trasplante de Hígado; Trasplante de Células Madre Hematopoyéticas; Trasplante de Riñón; Cuidado Dental; Radiografía Panorámica. 
Uso de radiografias panorâmicas no programa de assistência odontológica a pacientes de transplante da UFMG

\section{INTRODUÇÃO}

A radiografia panorâmica proporciona uma visão ampla das estruturas dentoalveolares, sendo útil para a avaliação do estado do osso nos maxilares, oferecendo uma visão de toda a dentição e estruturas ósseas de uma só vez (ABDINIAN et al., 2019).

O Programa de Extensão Assistência Odontológica a Pacientes de Transplante da UFMG - PAOPT - (que atende pacientes em fase anterior e posterior ao transplante de medula óssea, fígado e rins) funciona desde 2002. A partir de 2016, a realização de radiografias panorâmicas faz parte do protocolo para o diagnóstico e planejamento da atenção, baseado nas recomendações da American Dental Association (ADA, 2012). Este método de diagnóstico por imagem traz vantagens como ampla visibilidade dos ossos faciais e dentes; baixa dose de radiação e não causa desconforto ao paciente, que muitas vezes já apresenta lesões na mucosa bucal em função do preparo para o transplante ou da medicação pós-transplante (MIRANDA et al., 2016). Radiografias periapicais e interproximais são requeridas, mas a prévia análise da panorâmica já permite o planejamento inicial e indicação mais precisa das demais radiografias (ADA, 2012). Inspeções visuais não são suficientes para detectar várias doenças bucais que afetam o tecido subgengival ou são escondidas pelo esmalte dentário (KWEON et al., 2018). Embora um estudo radiográfico de boca toda seja superior a uma radiografia panorâmica, ambos os exames podem ser necessários para maximizar a quantidade de informação radiográfica disponível antes da realização de um transplante de medula ou sobre pacientes que possuem falência crônica hepática que esperam por um transplante, por exemplo (BISHAY et al., 1999; GHAPANCHI et al., 2018).

O objetivo do presente estudo é caracterizar os pacientes e levantar aspectos radiográficos relevantes observados nas radiografias panorâmicas do PAOPT. Este é um projeto de extensão que envolve o trabalho conjunto das Faculdades de Odontologia e Medicina da UFMG. Nele, pacientes que estão na fase pré e pós transplante recebem cuidado odontológico integral com a finalidade de promover a saúde bucal deles, refletindo em sua saúde geral. Além do trabalho interdisciplinar entre odontologia, medicina e enfermagem, as diversas áreas da odontologia como radiologia, periodontia, clínica, endodontia, prótese e estomatologia, ajudam a embasar a forte característica de atenção multiprofissional que garante a integralidade do cuidado ao paciente oncológico.

A experiência deste projeto pretende auxiliar outros projetos interdisciplinares semelhantes, na organização dos estudos preliminares de planejamento integral do tratamento odontológico a essa parcela da população. Com isso, pretende-se cumprir as diretrizes da extensão universitária de indissociabilidade ensino/pesquisa/extensão e de impacto e transformação social, uma vez que 
Uso de radiografias panorâmicas no programa de assistência odontológica a pacientes de transplante da UFMG esta experiência exitosa pode auxiliar em outras realidades semelhantes no Brasil, trazendo maior rapidez no tratamento odontológico pré-transplante.

\section{MÉTODOS}

Foram estudadas radiografias panorâmicas dos pacientes do PAOPT, por dois avaliadores (LPPA e JAB) previamente treinados e calibrados que desconheciam a história clínica do paciente. Esse treinamento e essa calibração foram feitos tendo por base exercícios de análises de radiografias panorâmicas. Quando os examinadores discordavam entre si, um terceiro avaliador, professor de radiologia odontológica (TMPA) realizava o exame. Duas semanas depois os exames foram repetidos para obtenção dos valores de Kappa intraexaminador. Os valores do índice Kappa interexaminador variaram de 0,75 a 0,78 e os valores intraexaminadores variaram de 0,77 a 0,80 (LPPA) e 0,78 a 0,80 (JAB) para os itens investigados. Esses resultados da calibração foram considerados muito bons (MIOT, 2016) e a pesquisa prosseguiu.

Todas as imagens panorâmicas foram obtidas com a mesma máquina e a correção de ampliação padrão não foi aplicada, considerando que a taxa de ampliação pode ser variável entre diferentes partes da mesma radiografia, dependendo no posicionamento do paciente.

Esta pesquisa foi submetida à análise do Comitê de Ética em Pesquisa em Humanos sendo aprovada com o número CAAE:77375517.9.0000.5149.

A análise estatística se deu por análise descritiva dos resultados encontrados por percentuais, médias e desvios-padrão. Também foram usadas análises bivariadas empregando-se o teste do qui-quadrado e teste exato de Fischer, teste de Mann-Whitney para comparação de grupos pré e pós tratamento odontológico e teste de Kruskal-Wallis. Todos os resultados foram considerados significativos para uma probabilidade de significância inferior a 5\% ( $p<0,05)$, tendo, portanto, pelo menos $95 \%$ de confiança nas conclusões apresentadas.

\section{RESULTADOS}

Participaram desse estudo radiografias panorâmicas de 182 pacientes, 64,3\% do sexo masculino. Entre os pacientes, 2,2\% tinham menos de 18 anos; 19,4\% de 18 a 39 anos; 49,7\% de 40 a 59 anos e $28,7 \%$ tinham pelo menos 60 anos. A maioria $(53,1 \%)$ foi submetida ao transplante de fígado, 37\% de medula óssea e 9,9\% de rins. Cerca de $84 \%$ dos pacientes foram avaliados na fase pré-transplante e possuíam em média 21,1 dentes presentes. (Tabela 1). 
Uso de radiografias panorâmicas no programa de assistência odontológica a pacientes de transplante da UFMG

Tabela 1: Caracterização das variáveis de interesse

\begin{tabular}{|c|c|c|}
\hline \multirow{2}{*}{ Característica } & \multicolumn{2}{|l|}{ Frequência } \\
\hline & $\mathrm{N}$ & $\%$ \\
\hline \multicolumn{3}{|l|}{ Sexo } \\
\hline Feminino & 65 & 35,7 \\
\hline Masculino & 117 & 64,3 \\
\hline Total & 182 & 100,0 \\
\hline Idade & $49,6 \pm 14,7$ & \\
\hline \multicolumn{3}{|l|}{ Faixa etária } \\
\hline$<18$ anos & 4 & 2,2 \\
\hline 18 a 39 anos & 35 & 19,4 \\
\hline 40 a 59 anos & 90 & 49,7 \\
\hline 60 anos ou mais & 52 & 28,7 \\
\hline Total & $181 *$ & 100,0 \\
\hline \multicolumn{3}{|l|}{ Tipo de transplante } \\
\hline Fígado & 96 & 53,1 \\
\hline Medula óssea & 67 & 37,0 \\
\hline Rins & 18 & 9,9 \\
\hline Total & $181 *$ & 100,0 \\
\hline \multicolumn{3}{|l|}{ Fase do transplante } \\
\hline Pós-Transplante & 30 & 16,6 \\
\hline Pré-transplante & 151 & 83,4 \\
\hline Total & $181 *$ & 100,0 \\
\hline $\mathrm{N}^{\mathrm{o}}$ total de dentes & $21,1 \pm 8,5$ & \\
\hline $\mathrm{N}^{\mathrm{o}}$ total de dentes na maxila & $10,3 \pm 4,9$ & \\
\hline $\mathrm{N}^{\mathrm{o}}$ total de dentes na mandibula & $10,8 \pm 4,1$ & \\
\hline
\end{tabular}

Nota: Base de dados: 182 radiografias panorâmicas de pacientes do PAOPT. Um prontuário não apresentou dados sobre idade, tipo e fase pré ou pós transplante

A Tabela 2 mostra uma caracterização dos pacientes em relação às variáveis de interesse, considerando-se a avaliação da maxila, da mandíbula e de uma forma global.

Tabela 2: Caracterização das variáveis de interesse considerando-se a maxila, a mandíbula e o global

\begin{tabular}{|c|c|c|c|c|c|c|}
\hline \multirow{3}{*}{ Característica } & \multicolumn{4}{|c|}{ Região } & \multirow{2}{*}{\multicolumn{2}{|c|}{ Global }} \\
\hline & \multicolumn{2}{|c|}{ Maxila } & \multicolumn{2}{|c|}{ Mandíbula } & & \\
\hline & $\mathrm{n}$ & $\%$ & $\mathrm{~N}$ & $\%$ & $\mathrm{n}$ & $\%$ \\
\hline \multicolumn{7}{|l|}{ Tipo de trabeculagem } \\
\hline Homogêneo & 165 & 90,7 & 148 & 81,3 & 138 & 75,8 \\
\hline Heterogêneo & 17 & 9,3 & 34 & 18,7 & 44 & 24,2 \\
\hline \multicolumn{7}{|l|}{ Dente intraósseo } \\
\hline Ausente & 162 & 89,0 & 167 & 91,8 & 163 & 89,6 \\
\hline
\end{tabular}


Uso de radiografias panorâmicas no programa de assistência odontológica a pacientes de transplante da UFMG
Presente
20
$11,0 \quad 15$
8,2
19
10,4

Raiz residual

Ausente

Presente

Lesão radiopaca

Ausente

Lesão radiolúcida

Ausente

Enxerto ósseo

Ausente

Reabsorção radicular

$$
\begin{aligned}
& \text { Ausente } \\
& \text { Presente }
\end{aligned}
$$

Nível ósseo

Normal
Leve
Moderado
Severo

Lesão periapcal

$$
\text { Ausente }
$$

Prótese fixa

$$
\text { Ausente }
$$

Presença de implante

Ausente
Presente

Tratamento endodôntico

Ausente

Seio maxilar

$$
\text { Radiolúcido }
$$

Relação da cúpula alveolar

$$
\begin{aligned}
& \text { Ausente } \\
& \text { Presente }
\end{aligned}
$$

$\begin{array}{llllll}154 & 84,6 & 162 & 89,0 & 146 & 80,2 \\ 28 & 15,4 & 20 & 11,0 & 36 & 19,8\end{array}$

$\begin{array}{llllll}181 & 99,5 & 175 & 96,2 & 174 & 95,6 \\ 1 & 0,5 & 7 & 3,8 & 8 & 4,4\end{array}$

$\begin{array}{llllll}175 & 96,2 & 175 & 96,2 & 170 & 93,4 \\ 7 & 3,8 & 7 & 3,8 & 12 & 6,6\end{array}$

$\begin{array}{llllll}182 & 100,0 & 182 & 100,0 & 182 & 100,0 \\ 0 & 0,0 & 0 & 0,0 & 0 & 0,0\end{array}$

$\begin{array}{llllll}171 & 94,0 & 175 & 96,2 & 168 & 92,3 \\ 11 & 6,0 & 7 & 3,8 & 14 & 7,7\end{array}$

$\begin{array}{llllll}14 & 7,7 & 11 & 6,0 & 2 & 1,1 \\ 99 & 54,4 & 105 & 57,7 & 88 & 48,4 \\ 57 & 31,3 & 61 & 33,5 & 77 & 42,3 \\ 12 & 6,6 & 5 & 2,7 & 15 & 8,2\end{array}$

$\begin{array}{llllll}154 & 84,6 & 156 & 85,7 & 142 & 78,0 \\ 28 & 15,4 & 26 & 14,3 & 40 & 22,0\end{array}$

$\begin{array}{llllll}134 & 73,6 & 154 & 84,6 & 130 & 71,4 \\ 48 & 26,4 & 28 & 15,4 & 52 & 28,6\end{array}$

$\begin{array}{llllll}175 & 96,2 & 179 & 98,4 & 174 & 95,6 \\ 7 & 3,8 & 3 & 1,6 & 8 & 4,4\end{array}$

$\begin{array}{llllll}113 & 62,1 & 140 & 76,9 & 107 & 58,8 \\ 69 & 37,9 & 42 & 23,1 & 75 & 41,2\end{array}$

$\begin{array}{llllll}134 & 73,6 & - & - & 134 & 73,6 \\ 48 & 26,4 & - & - & 48 & 26,4 \\ & & & & & \\ 177 & 97,3 & - & - & 177 & 97,3 \\ 5 & 2,7 & - & - & 5 & 2,7\end{array}$

Base de dados: 182 radiografias panorâmicas de pacientes do PAOPT 
A Tabela 3 mostra uma avaliação da associação da faixa etária, tipo de transplante e fase do tratamento com as variáveis de interesse. Nenhuma associação significativa foi observada entre as variáveis de interesse com tipo de transplante e fase do tratamento. Em relação à faixa etária foi observado uma associação significativa com a avaliação da raiz residual, nível ósseo e relação da cúpula alveolar.

Tabela 3: Avaliação da associação da faixa etária, tipo de transplante e fase do tratamento com variáveis de interesse

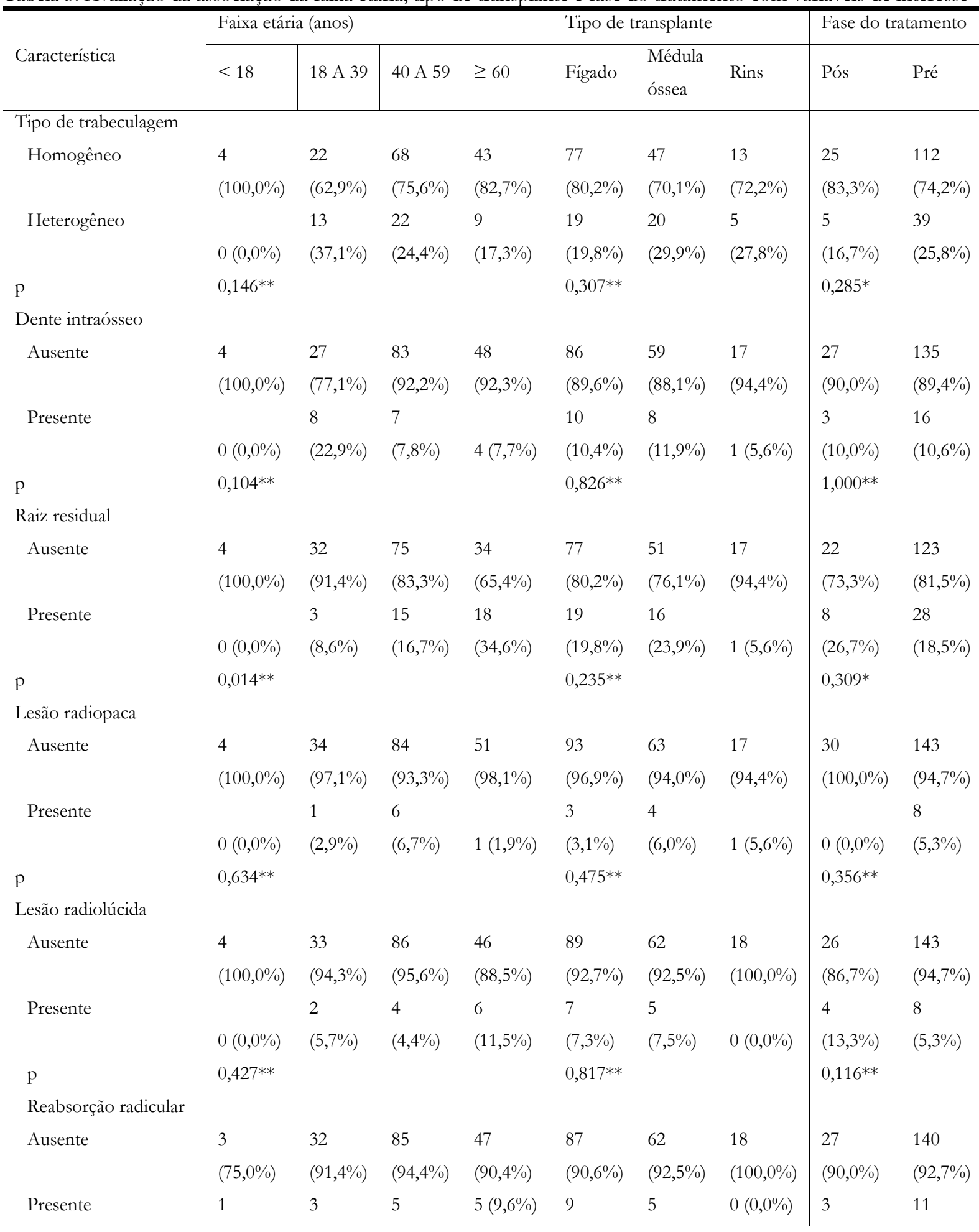




\begin{tabular}{|c|c|c|c|c|c|c|c|c|c|}
\hline $\begin{array}{l}\mathrm{p} \\
\text { Nível ósseo }\end{array}$ & $\begin{array}{l}(25,0 \%) \\
0,347 * *\end{array}$ & $(8,6 \%)$ & $(5,6 \%)$ & & $\begin{array}{l}(9,4 \%) \\
0,505^{* *}\end{array}$ & $(7,5 \%)$ & & $\begin{array}{l}(10,0 \%) \\
0,706^{* *}\end{array}$ & $7,3 \%)$ \\
\hline Normal & $0(0,0 \%)$ & $\begin{array}{l}0 \\
(0,0 \%)\end{array}$ & $\begin{array}{l}1 \\
(1,1 \%)\end{array}$ & $1(1,9 \%)$ & $\begin{array}{l}2 \\
(2,1 \%)\end{array}$ & $\begin{array}{l}0 \\
(0,0 \%)\end{array}$ & $0(0,0 \%)$ & $0(0,0 \%)$ & $\begin{array}{l}2 \\
(1,3 \%)\end{array}$ \\
\hline Perda Leve & $\begin{array}{l}2 \\
(50,0 \%)\end{array}$ & $\begin{array}{l}26 \\
(74,3 \%)\end{array}$ & $\begin{array}{l}44 \\
(48,9 \%)\end{array}$ & $\begin{array}{l}16 \\
(30,8 \%)\end{array}$ & $\begin{array}{l}44 \\
(45,8 \%)\end{array}$ & $\begin{array}{l}37 \\
(55,2 \%)\end{array}$ & $\begin{array}{l}7 \\
(38,9 \%)\end{array}$ & $\begin{array}{l}16 \\
(53,3 \%)\end{array}$ & $\begin{array}{l}72 \\
(47,7 \%)\end{array}$ \\
\hline Perda Moderada & $\begin{array}{l}2 \\
(50,0 \%)\end{array}$ & $\begin{array}{l}8 \\
(22,9 \%)\end{array}$ & $\begin{array}{l}36 \\
(40,0 \%)\end{array}$ & $\begin{array}{l}30 \\
(57,7 \%)\end{array}$ & $\begin{array}{l}42 \\
(43,8 \%)\end{array}$ & $\begin{array}{l}25 \\
(37,3 \%)\end{array}$ & $9(50 \%)$ & $\begin{array}{l}12 \\
(40,0 \%)\end{array}$ & $\begin{array}{l}64 \\
(42,4 \%)\end{array}$ \\
\hline Perda Severa & $0(0,0 \%)$ & $\begin{array}{l}1 \\
(2,9 \%)\end{array}$ & $\begin{array}{l}9 \\
(10,0 \%)\end{array}$ & $5(9,6 \%)$ & $\begin{array}{l}8 \\
(8,3 \%)\end{array}$ & $\begin{array}{l}5 \\
(7,5 \%)\end{array}$ & $\begin{array}{l}2 \\
(11,1 \%)\end{array}$ & $2(6,7 \%)$ & $\begin{array}{l}13 \\
(8,6 \%)\end{array}$ \\
\hline $\begin{array}{l}\mathrm{p} \\
\text { Lesão periapical }\end{array}$ & $0,016^{* *}$ & & & & $0,733^{* *}$ & & & & $0,968^{* *}$ \\
\hline Ausente & $\begin{array}{l}3 \\
(75,0 \%)\end{array}$ & $\begin{array}{l}30 \\
(85,7 \%)\end{array}$ & $\begin{array}{l}70 \\
(77,8 \%)\end{array}$ & $\begin{array}{l}39 \\
(75,0 \%)\end{array}$ & $\begin{array}{l}74 \\
(77,1 \%)\end{array}$ & $\begin{array}{l}56 \\
(83,6 \%)\end{array}$ & $\begin{array}{l}12 \\
(66,7 \%)\end{array}$ & $\begin{array}{l}22 \\
(73,3 \%)\end{array}$ & $\begin{array}{l}120 \\
(79,5 \%)\end{array}$ \\
\hline Presente & $\begin{array}{l}1 \\
(25,0 \%)\end{array}$ & $\begin{array}{l}5 \\
(14,3 \%)\end{array}$ & $\begin{array}{l}20 \\
(22,2 \%)\end{array}$ & $\begin{array}{l}13 \\
(25,0 \%)\end{array}$ & $\begin{array}{l}22 \\
(22,9 \%)\end{array}$ & $\begin{array}{l}11 \\
(16,4 \%)\end{array}$ & $\begin{array}{l}6 \\
(33,3 \%)\end{array}$ & $\begin{array}{l}8 \\
(26,7 \%)\end{array}$ & $\begin{array}{l}31 \\
(20,5 \%)\end{array}$ \\
\hline $\begin{array}{l}\mathrm{p} \\
\text { Prótese fixa }\end{array}$ & $0,644 * *$ & & & & $0,273^{* *}$ & & & $0,455^{*}$ & \\
\hline Ausente & $\begin{array}{l}3 \\
(75,0 \%)\end{array}$ & $\begin{array}{l}25 \\
(71,4 \%)\end{array}$ & $\begin{array}{l}59 \\
(65,6 \%)\end{array}$ & $\begin{array}{l}42 \\
(80,8 \%)\end{array}$ & $\begin{array}{l}70 \\
(72,9 \%)\end{array}$ & $\begin{array}{l}46 \\
(68,7 \%)\end{array}$ & $\begin{array}{l}13 \\
(72,2 \%)\end{array}$ & $\begin{array}{l}22 \\
(73,3 \%)\end{array}$ & $\begin{array}{l}107 \\
(70,9 \%)\end{array}$ \\
\hline Presente & $\begin{array}{l}1 \\
(25,0 \%)\end{array}$ & $\begin{array}{l}10 \\
(28,6 \%)\end{array}$ & $\begin{array}{l}31 \\
(34,4 \%)\end{array}$ & $\begin{array}{l}10 \\
(19,2 \%)\end{array}$ & $\begin{array}{l}26 \\
(27,1 \%)\end{array}$ & $\begin{array}{l}21 \\
(31,3 \%)\end{array}$ & $\begin{array}{l}5 \\
(27,8 \%)\end{array}$ & $\begin{array}{l}8 \\
(26,7 \%)\end{array}$ & $\begin{array}{l}44 \\
(29,1 \%)\end{array}$ \\
\hline $\begin{array}{l}\mathrm{P} \\
\text { Presença de implante }\end{array}$ & $0,271 * *$ & & & & $0,836^{*}$ & & & $0,785^{*}$ & \\
\hline Ausente & $\begin{array}{l}4 \\
(100,0 \%)\end{array}$ & $\begin{array}{l}34 \\
(97,1 \%)\end{array}$ & $\begin{array}{l}85 \\
(94,4 \%)\end{array}$ & $\begin{array}{l}50 \\
(96,2 \%)\end{array}$ & $\begin{array}{l}94 \\
(97,9 \%)\end{array}$ & $\begin{array}{l}62 \\
(92,5 \%)\end{array}$ & $\begin{array}{l}17 \\
(94,4 \%)\end{array}$ & $\begin{array}{l}27 \\
(90,0 \%)\end{array}$ & $\begin{array}{l}146 \\
(96,7 \%)\end{array}$ \\
\hline Presente & $0(0,0 \%)$ & $\begin{array}{l}1 \\
(2,9 \%)\end{array}$ & $\begin{array}{l}5 \\
(5,6 \%)\end{array}$ & $2(3,8 \%)$ & $\begin{array}{l}2 \\
(2,1 \%)\end{array}$ & $\begin{array}{l}5 \\
(7,5 \%)\end{array}$ & $1(5,6 \%)$ & $\begin{array}{l}3 \\
(10,0 \%)\end{array}$ & $\begin{array}{l}5 \\
(3,3 \%)\end{array}$ \\
\hline $\begin{array}{l}\mathrm{p} \\
\text { Tratamento } \\
\text { endodôntico }\end{array}$ & $1,000^{* *}$ & & & & $0,170^{* *}$ & & & $0,129 * *$ & \\
\hline Ausente & $\begin{array}{l}3 \\
(75,0 \%)\end{array}$ & $\begin{array}{l}21 \\
(60,0 \%)\end{array}$ & $\begin{array}{l}50 \\
(55,6 \%)\end{array}$ & $\begin{array}{l}33 \\
(63,5 \%)\end{array}$ & $\begin{array}{l}63 \\
(65,6 \%)\end{array}$ & $\begin{array}{l}36 \\
(53,7 \%)\end{array}$ & $\begin{array}{l}8 \\
(44,4 \%)\end{array}$ & $\begin{array}{l}17 \\
(56,7 \%)\end{array}$ & $\begin{array}{l}90 \\
(59,6 \%)\end{array}$ \\
\hline Presente & $\begin{array}{l}1 \\
(25,0 \%)\end{array}$ & $\begin{array}{l}14 \\
(40,0 \%)\end{array}$ & $\begin{array}{l}40 \\
(44,4 \%)\end{array}$ & $\begin{array}{l}19 \\
(36,5 \%)\end{array}$ & $\begin{array}{l}33 \\
(34,4 \%)\end{array}$ & $\begin{array}{l}31 \\
(46,3 \%)\end{array}$ & $\begin{array}{l}10 \\
(55,6 \%)\end{array}$ & $\begin{array}{l}13 \\
(43,3 \%)\end{array}$ & $\begin{array}{l}61 \\
(40,4 \%)\end{array}$ \\
\hline $\begin{array}{l}\mathrm{p} \\
\text { Seio maxilar }\end{array}$ & $0,754^{* *}$ & & & & $0,132^{*}$ & & & & $0,765^{*}$ \\
\hline Radiolúcido & $\begin{array}{l}1 \\
(25,0 \%)\end{array}$ & $\begin{array}{l}25 \\
(71,4 \%)\end{array}$ & $\begin{array}{l}66 \\
(73,3 \%)\end{array}$ & $\begin{array}{l}42 \\
(80,8 \%)\end{array}$ & $\begin{array}{l}69 \\
(71,9 \%)\end{array}$ & $\begin{array}{l}49 \\
(73,1 \%)\end{array}$ & $\begin{array}{l}16 \\
(88,9 \%)\end{array}$ & $\begin{array}{l}19 \\
(63,3 \%)\end{array}$ & $\begin{array}{l}115 \\
(76,2 \%)\end{array}$ \\
\hline CMR & $\begin{array}{l}3 \\
(75,0 \%)\end{array}$ & $\begin{array}{l}10 \\
(28,6 \%)\end{array}$ & $\begin{array}{l}24 \\
(26,7 \%)\end{array}$ & $\begin{array}{l}10 \\
(19,2 \%)\end{array}$ & $\begin{array}{l}27 \\
(28,1 \%)\end{array}$ & $\begin{array}{l}18 \\
(26,9 \%)\end{array}$ & $\begin{array}{l}2 \\
(11,1 \%)\end{array}$ & $\begin{array}{l}11 \\
(36,7 \%)\end{array}$ & $\begin{array}{l}36 \\
(23,8 \%)\end{array}$ \\
\hline $\begin{array}{l}\text { P } \\
\text { Relação da cúpula } \\
\text { alveolar }\end{array}$ & $0,110^{* *}$ & & $*$ & & $0,333^{* *}$ & & & $0,143^{*}$ & \\
\hline Ausente & 3 & 33 & 88 & 52 & 93 & 65 & 18 & 30 & 146 \\
\hline
\end{tabular}


Uso de radiografias panorâmicas no programa de assistência odontológica a pacientes de transplante da UFMG

\begin{tabular}{l|llll|lll|lll} 
& $(75,0 \%)$ & $(94,3 \%)$ & $(97,8 \%)$ & $(100,0 \%)$ & $(96,9 \%)$ & $(97 \%)$ & $(100,0 \%)$ & $(100,0 \%)$ & $(96,7 \%)$ \\
Presente & 1 & 2 & 2 & & 3 & & & & 5 \\
& & $(25,0 \%)$ & $(5,7 \%)$ & $(2,2 \%)$ & $0(0,0 \%)$ & $(3,1 \%)$ & $2(3 \%)$ & $0(0,0 \%)$ & $0(0,0 \%)$ & $(3,3 \%)$ \\
$\mathrm{p}$ & $0,041 * *$ & & & & & $1,000 * *$ & & $0,592^{* *}$ \\
\hline
\end{tabular}

Base de dados: 181 radiografias panorâmicas de pacientes do PAOPT; Nota: a probabilidade de significância refere-se ao Qui-quadrdo $(*)$ ou teste exato de Fisher(**)

Em relação à presença de raiz residual, observou-se um aumento à medida que a idade dos pacientes foi aumentando. Nenhum dos pacientes com menos de 18 anos apresentou uma raiz residual; de 18 a 39 este percentual foi de 8,6\%; no grupo de 40 a 59 anos foi de 16,7\%, e no grupo com pelo menos 60 anos foi de 34,6\% (Tabela 3).

Em relação ao nível ósseo, observou-se um aumento dos pacientes com perdas moderadas ou severas do nível ósseo, sendo que no grupo de 18 a 39 anos observou-se 25,8\% de pacientes com essa característica; no grupo de 40 a 59 anos este percentual foi igual a 50\%, e no grupo com 60 anos ou mais este percentual ultrapassa os $65 \%$ de casos. No grupo mais jovem observou-se $50 \%$ de pacientes com perda leve no nível ósseo e 50\% com perda moderada no nível ósseo. Vale ressaltar que neste grupo temos apenas 4 pacientes (Tabela 3).

O percentual de pacientes com uma relação da cúpula alveolar presente foi reduzindo à medida que a idade dos pacientes aumentou. No grupo mais jovem, 25\% dos pacientes tinham presente a relação da cúpula alveolar; no grupo de 18 a 39 anos este percentual foi de 5,7\%; no grupo de 40 a 59 anos foi de $2,2 \%$ e não foi observado nenhum paciente com esta característica no grupo mais velho (Tabela 3).

Em relação ao Índice de Dentes Cariados, Perdidos e Obturados (CPOd) foram identificadas diferenças significativas apenas quando compararam-se os grupos de faixa etária, sendo que o grupo com idade entre 18 e 39 não apresentou diferenças significativas em relação ao grupo de 40 a 59 anos e ambos apresentaram um valor de CPOd significativamente inferior ao observado no grupo com 60 anos ou mais. Neste estudo não foram considerados os pacientes com até 18 anos por se tratar de um grupo muito reduzido. Não foi identificada uma influência significativa do tipo de transplante e da fase do tratamento na avaliação do CPOd (Tabela 4). 
Uso de radiografias panorâmicas no programa de assistência odontológica a pacientes de transplante da UFMG

Tabela 4: Avaliação da influência da faixa etária, tipo de transplante e fase do tratamento no CPOd

\begin{tabular}{|c|c|c|c|c|c|}
\hline \multirow{2}{*}{ Variáveis } & \multicolumn{4}{|c|}{ Medidas descritivas } & \multirow{2}{*}{$\mathrm{P}$} \\
\hline & Mínimo & Máximo & Mediana & $\bar{x} \pm d p$ & \\
\hline \multicolumn{6}{|l|}{ Faixa etária } \\
\hline$<18$ & 0 & 6 & 1,0 & $2,3 \pm 3,2$ & \multirow{4}{*}{$\begin{array}{l}<0,001^{*} \\
\left(\mathrm{~F}_{2}=\mathrm{F}_{3}\right)>\mathrm{F}_{4}\end{array}$} \\
\hline 18 a $39^{\mathrm{F} 2}$ & 0 & 18 & 7,5 & $8,8 \pm 5,9$ & \\
\hline 40 a $59^{\mathrm{F} 3}$ & 0 & 28 & 18,5 & $17,1 \pm 6,8$ & \\
\hline 60 anos ou mais ${ }^{\mathrm{F} 4}$ & 9 & 28 & 20,0 & $19,5 \pm 5,1$ & \\
\hline \multicolumn{6}{|l|}{ Tipo de transplante } \\
\hline Fígado $^{\mathrm{T} 1}$ & 0 & 28 & 16,0 & $15,9 \pm 7,8$ & $0,674^{*}$ \\
\hline Medula óssea ${ }^{\mathrm{T} 2}$ & 0 & 28 & 17,0 & $15,0 \pm 7,5$ & $\mathrm{~T} 1=\mathrm{T} 2=\mathrm{T} 3$ \\
\hline Rins $^{\mathrm{T} 3}$ & 3 & 23 & 18,0 & $16,8 \pm 5,6$ & \\
\hline \multicolumn{6}{|l|}{ Fase do tratamento } \\
\hline Pós & 0 & 27 & 15,0 & $14,1 \pm 8,5$ & $0,746^{* *}$ \\
\hline Pré & 0 & 28 & 17,0 & $15,9 \pm 7,3$ & Pós = Pré \\
\hline
\end{tabular}

Base de dados: 125 radiograficas panorâmicas de pacientes do PAOPT

Nota: a probabilidade de significância refere-se ao teste Kruskal-Waliss $\left(^{*}\right)$ ou teste de MannWhitney $(* *)$

\section{DISCUSSÃO}

Neste estudo, observou-se uma maior prevalência de dentes cariados, perdidos ou obturados na faixa etária de 60 anos ou mais. O sexo masculino foi mais prevalente do que o sexo feminino.

A variação de faixa etária foi menor do que aquele estudo realizado com pacientes à espera de transplante de fígado no Irã (GHAPANCHI et al.,2018), e com variação etária maior àquela apresentada no estudo com pacientes com doença renal crônica iranianos (ABDINIAN et al., 2019).

A média de dentes examinados é semelhante à apresentada num estudo prospectivo realizado em Helsinke, Finlândia, entre pacientes pré e pós-transplante renal (RUOKONEN et al., 2019).

Foi encontrada uma prevalência de $12 \%$ de periodontite entre indivíduos em fase prétransplante de fígado (GHAPANCHI et al., 2018) e uma prevalência de 20\% de perda óssea grave entre indivíduos à espera de transplante de células hematopoiéticas (BISHAY et al., 1999). Entre indivíduos típicos, a perda óssea marginal (não há menção se grave ou moderada ou ambas) e as lesões de furca foram detectadas em $64,1 \%$ e 38,4\% dos exames radiológicos panorâmicos, respectivamente, também se empregando análise de radiografias panorâmicas (KWEON et al., 
Uso de radiografias panorâmicas no programa de assistência odontológica a pacientes de transplante da UFMG

2018). Neste estudo não se apresentam os dados relativos ao exame de sondagem periodontal. $O$ percentual encontrado no presente estudo sobre perda óssea grave foi de 8,3\% (pré- transplante de fígado) e 7,5\% pré-transplante de medula. As diferenças entre os resultados podem ser devido à variação maior de faixa etária das pessoas nos estudos investigados (KWEON et al., 2018; GHAPANCHI et al., 2018).

Não foram observadas diferenças estatisticamente significativas entre indivíduos pré e póstransplante de forma global. Entretanto, em um estudo longitudinal, entre indivíduos pré e pós transplante, foram detectadas diferenças entre as profundidades de sondagem, sendo que as medidas mais profundas foram detectadas no grupo pré-transplante renal (NYLUND et al., 2018). As diferenças entre os dois estudos reside no desenho: o presente estudo é transversal, e na forma com a qual os dados foram coletados: no caso do estudo longitudinal, a sondagem também foi incluída (NYLUND et al., 2018). Curiosamente, entre pacientes que receberam transplante de fígado, a comparação entre as radiografias panorâmicas indica uma perda óssea após 10 anos de acompanhamento com significado estatístico. Observou-se uma perda anual de aproximadamente $0,11 \mathrm{~mm}$. Os autores ponderam que esta perda está mais relacionada ao avanço da idade do que propriamente à condição sistêmica dos pacientes (MACHTEI et al., 2012).

Dentes perdidos foram detectados em 59,2\% das radiografias panorâmicas no estudo realizado na Coréia, com indivíduos típicos (KWEON et al., 2018). No presente estudo, raízes residuais foram detectadas em 19,8\% dos exames radiológicos. Tais resultados podem ser explicados pela metodologia empregada entre os dois estudos. O estudo coreano emprega o termo “dentes perdidos”, que podem ser observados também pela inspeção visual.

Foram encontradas em estudo anterior $27,5 \%$ de lesões radiopacas e $11,6 \%$ de lesões radiolúcidas entre aqueles que esperam um transplante de fígado (GHAPANCHI et al., 2018). Nossos resultados foram de 3,1\% de lesões radiopacas e 7,3\% de lesões radiolúcidas dentre pacientes pré-transplante de fígado. No caso das lesões radiolúcidas, a explicação se relaciona ao método de coleta de dados. No estudo iraniano, as lesões radiolúcidas foram agrupadas às lesões periapicais, o que não foi realizado pelo presente estudo. Entretanto, não foram encontradas explicações plausíveis na literatura consultada para a grande diferença entre os percentuais encontrados para as lesões radiopacas.

Em estudo anterior, não foram encontraram diferenças entre perda óssea e doenças renais crônicas, mas defeitos no córtex mandibular foram apontados com maior frequência nos pacientes caso do que entre os controles (ABDINIAM et al., 2019). No presente estudo, quando são comparados os grupos de pré e pós-transplantados de fígado, rins e células hematopoiéticas, não 
Uso de radiografias panorâmicas no programa de assistência odontológica a pacientes de transplante da UFMG

foram encontradas diferenças em termos de trabeculagem óssea, perda no nível ósseo alveolar e lesões radiopacas.

Em relação à presença de próteses fixas, o percentual apresentado pelos pacientes do PAOPT é superior àquele apresentado para o Brasil no SB 2010 (BRASIL, 2012), o que pode indicar que esta população tem acesso a tratamentos odontológicos mais complexos, como comprovado também pelos resultados relativos a tratamentos endodônticos e implantes.

Este estudo é inédito porque, até o presente momento, nenhuma pesquisa descreveu a prevalência de anormalidades em estruturas ósseas e subgengivais entre indivíduos que esperam um transplante de medula, fígado ou rim ou que já foram submetidos ao procedimento. Ainda assim, esta pesquisa possui algumas limitações como a tomada radiográfica por vários operadores.

É preciso considerar, entretanto, que o objetivo principal da tomada radiográfica panorâmica neste projeto de extensão é acelerar o diagnóstico, a elaboração e a execução do planejamento integral do paciente do PAOPT. Além disso, muitas vezes os pacientes que aguardam transplantes apresentam lesões de mucosa, resultado do uso de medicamentos, que inviabilizam as tomadas radiográficas periapicais e interproximais. Esta agilidade é necessária, pois quando o órgão compatível é identificado o tratamento odontológico pode ser suspenso abruptamente. Não há tempo a perder. A equipe de transplante não pode esperar pela resolução dos problemas odontológicos, por isso é que o trabalho interdisciplinar deve estar concatenado para garantir o sucesso do transplante. Apesar de as tomadas radiográficas serem realizadas pelo setor de radiologia da Faculdade de Odontologia da UFMG, tem-se que considerar que o objetivo primeiro não é gerar estudos científicos e, sim, contribuir positivamente com o impacto e a transformação social. Entretanto, a partir de dados gerados pelos serviços, muito se pode avançar na compreensão de problemas epidemiológicos que afetam grupos ou a população em geral. Com isso, pretende-se acançar as diretrizes de indissociabilidade do ensino/pesquisa/extensão e também da relação dialógica com a sociedade que aqui é traduzida pelas diversas equipes odontológicas no Brasil, as quais trabalham conjuntamente com a equipe de transplante para garantir uma melhor qualidade de vida a essa população.

Em relação à extensão universitária, este é um trabalho de colaboração entre duas Faculdades da UFMG. Seu objetivo principal é estabilizar a saúde bucal do paciente em fase pré transplante, para que o mesmo possa ser submetido ao procedimento de forma segura. A vivência desta experiência pelo graduando em odontologia é ímpar. $O$ aluno aprende a construir o planejamento integral dentro dos limites impostos pelo tempo. Este é um projeto de extensão que possui várias experiências exitosas que podem e devem ser compartilhadas com outras instituições de ensino do país. Esse olhar cuidadoso para a divulgação de resultados é uma forma de estabelecer 
Uso de radiografias panorâmicas no programa de assistência odontológica a pacientes de transplante da UFMG

uma relação dialógica com a sociedade (no caso, com os cirurgiões-dentistas). A experiência de sucesso com radiografias panorâmicas, ao acelerar a implementação de um plano de tratamento integral ao paciente, impacta positivamente não só a saúde bucal deste, como também ajuda na segurança do procedimento do transplante,

\section{CONCLUSÃO}

As tomadas radiográficas panorâmicas possuem um importante papel de acelerar a elaboração do plano de tratamento, especialmente entre pacientes que estejam na fase prétransplante de fígado, rins ou células hematopoiéticas, os quais muitas vezes apresentam lesões de mucosa que inviabilizam radiografias periapicais e inteproximais. A rapidez da detecção de estruturas anômalas subgengivais ou intraósseas pode auxiliar as equipes de odontologia no estabelecimento de quais procedimentos devem ser priorizados num tratamento odontológico que se caracteriza pela agilidade na resolução de problemas, para que assim o paciente possa ser encaminhado com tranquilidade para o transplante e órgãos.

\section{REFERÊNCIAS}

ABDINIAN, M.; MORTAZAVI, M.; JANDAGHIAN Z. Comparison of skeletal changes related to patients with chronic kidney disease and healthy individuals in digital panoramic radiography. Indian J Dent Res, v.30, p.358-62, May- June, 2019. Disponível em: doi: 10.4103/ijdr.IJDR_175_18.

AMERICAN DENTAL ASSOCIATION Council on Scientific Affairs. U.S. DEPARTMENT OF HEALTH AND HUMAN SERVICES Public Health Service Food and Drug Administration. DENTAL RADIOGRAPHIC EXAMINATIONS: RECOMMENDATIONS FOR PATIENT SELECTION AND LIMITING RADIATION EXPOSURE, 2012. Disponível em: https://www.fda.gov/media/84818/download

BISHAY, N.; PETRIKOWSKI, C.G.; MAXYMIW, W.G.; LEE, L.; ABOP, D.; WOOD, R.E. Optimum dental radiography in bone marrow transplant patients. Optimum dental radiography in bone marrow transplant patients. Oral Surg Oral Med Oral Pathol Oral Radiol Endod, v. 87, p.375-9, March, 1999. Disponível em: doi: 10.1016/s1079-2104(99)70227-1.

BRASIL. Ministério da Saúde. SB Brasil 2010: Pesquisa Nacional de Saúde Bucal:

resultados principais. Brasília: Ministério da Saúde, 2012. Disponível em:

https://bvsms.saude.gov.br/bvs/publicacoes/pesquisa_nacional_saude_bucal.pdf

GHAPANCHI, J.; ZAHED, M.; HAGHNEGAHDAR, A.; NIAKAN, N.; SADEGHZADEH, A. Osteoporosis and Jaw Abnormalities in Panoramic Radiographyof Chronic Liver Failure Patients. BioMed Research International, v.26, p. 4280312, Aug. 2018. Disponível em: https://doi.org/10.1155/2018/4280312 
KWEON, H.H.; LEE, J.H; YOUK, T.; LEE, B.; KIM, H.T. Panoramic radiography can be an effective diagnostic tool adjunctive to oral examinations in the national health checkup program. Periodontal Implant Sci., v.48, n.5, p.317-325, Oct. 2018. Disponível em: doi: $10.5051 /$ jpis.2018.48.5.317

MACHTEI, E.E.; FALAH, M.; OETTINGER-BARAK, O.; BARUCH, Y.; HORWTIZ, J. Periodontal status in post-liver transplantation patients: 10 years of follow-up. Quintessence, v.43, n.10, p.879-885, Nov-Dec. 2012. Disponível em: https://pubmed.ncbi.nlm.nih.gov/23115767/

MIRANDA, S.S.; QUEIROZ, L.R.; FREITAS, V.S. Prevenção e tratamento das mucosites orais: uma revisão sistemática. Rev. Saúde Col. UEFS, v. 6, n.2, p. 66-73, Dezembro, 2016. Disponível em: http://periodicos.uefs.br/index.php/saudecoletiva/article/view/1189

MIOT, H.A. Análise de concordância em estudos clínicos e experimentaiS. J Vasc Bras. , v. 15, n.2, p.89-922016, Abr.-Jun. 2016. https://doi.org/10.1590/1677-5449.004216

NYLUND, K.M,; MEURMAN, J.H.; HEIKKINEN, A.M.; FURUHOLM, J.O.; ORTIZ, F.; RUOKONEN, H.M.Oral health in patients with renal disease:a longitudinal study from predialysis to kidney transplantation. Clin Oral Invest , v. 22, p.339-347, Jan. 2018. Disponível em: doi: 10.1007/s00784-017-2118-y

RUOKONEN, H.M.; NYLUND, K.; MEURMAN, J.H.; HEIKKINEN, A.M.; FURUHOLM, J.O.; SORSA ROINE, R.; ORTIZ, F.Oral symptoms and oral health-related quality of lifein patients with chronic kidney disease from predialysis to posttransplantation. Clinical Oral Investigations, v.23, p.2207-2213, May, 2019. Disponível em: doi: 10.1007/s00784-018-2647-z

Recebido em: 08/06/2021

Aceito em: 24/11/2021 